\title{
Application of the Richardson extrapolation in simulation studies of EEGs
}

\author{
$\begin{array}{ll}\text { J. W. H. Meijs } & \text { H. B. K. Boom }\end{array}$ \\ Department of Electrical Engineering, Twente University of Technology, POB 217, 7500 AE Enschede, \\ The Netherlands \\ M. J. Peters \\ Department of Technical Physics, Twente University of Technology, POB 217, 7500 AE Enschede, \\ The Netherlands
}

A. van Oosterom

University of Nijmegen, POB 9101, 6700 HB Nijmegen, The Netherlands

Keywords-EEG, Fredholm integral equation of the second kind, MEG, Multicompartment models, Neumann equation, Richardson extrapolation

Med. \& Biol. Eng. \& Comput., 1987, 25, 222-226

\section{Introduction}

TO BE ABLE to compute the potential distribution on the scalp due to brain activity (as reflected in EEGs) and the distribution of a component of the magnetic field (as reflected in MEGs), both the source and the volume conductor (i.e., the head) have to be modelled. Dipolar current sources are commonly used as a model for brain activity.

In the past it was common practice to describe the head by models for which an analytical solution exists. Usually, the head is described by a set of concentric spheres (Cuffin and Cohen, 1979; CoHen and Cuffin, 1983; ROMANI et al., 1985). Using this model, the active regions within the brains have been localised from the measured magnetic field data. These studies have been reviewed by OKADA (1983). The localisation of human focal epilepsy from measured magnetic field data is of clinical importance (SUTHERLING et al., 1984; BARTH et al. 1984; RicCI et al., 1984). Such a localisation will guide the neurosurgeon in those cases which are appropriate for surgical intervention. The use of a realistically shaped volume conductor model of the head (MeIJs et al., 1985), rather than a set of concentric spheres is not discussed in this paper. However, for this work we use the boundary element method for solving the associate forward problem of both the electrical potential and the magnetic field distribution. In the numerical computations some errors are to be expected in the approximate solution. These errors never proved to be a limitation in similar simulations of the cardiac electrical potential distribution and the cardiac magnetic field distribution (PETERs et al., 1983). Realistically chosen conduc-

Correspondence should be addressed to Ir.J.W. H. Meijs

First received 12th February and in final form 21st July 1986

(C) IFMBE: 1987 tivity ratios of the successive compartments for the torso model in the cardiac studies are of the order of $10: 1$, while for the head-model this ratio is of the order of $100: 1$. Because of this high conductivity ratio in the model of the head, the errors made in the numerical computation are magnified. In this paper this numerical problem will be described; it is solved by using the Richardson extrapolation method.

To study the numerical errors introduced by the discrete approximation of the surface integrals of the boundary element method, we restrict ourselves to concentric multispherical models as for these kinds of model, analytical expressions are available (CUFFIN and COHEN, 1979). We will focus on a model consisting of two concentric spheres, because in this case the errors in the approximate solution resulting from the discrete computations can be analysed and are of a similar nature to more elaborate multicompartment models.

The location of the source and the dimensions of both the spheres and the conductivity ratios are adapted to conform to the head. The source is located excentrical, simulating a source within the visual cortex. The conductivities chosen are equal to those of the skull and brain tissue.

\section{Basic equations}

The purpose of this section is to give the basic equations used for the solution of the potential distribution and the magnetic field distribution, produced by dipolar current sources embedded in a finite volume conductor consisting of a set of compartments. In each compartment the electrical conductivity has a constant value. The analytical equation for the potential distribution on each boundary 
surface $S_{l}$ within such a multi compartment model reads (BARNARD et al., 1967)

$$
V_{k}\left(\boldsymbol{r}^{\prime}\right)=\frac{\sigma_{s}}{\bar{\sigma}_{k}} V_{\infty}\left(\boldsymbol{r}^{\prime}\right)-\frac{1}{4 \pi \bar{\sigma}_{k}} \sum_{l} \Delta \sigma_{l} \int_{s_{l}} V_{l}(\boldsymbol{r}) d \Omega_{k l}
$$

with

$$
d \Omega_{k l}=\frac{\left(\boldsymbol{r}^{\prime}-\boldsymbol{r}^{\prime}\right)}{\left|\boldsymbol{r}^{\prime}-\boldsymbol{r}^{\prime}\right|^{3}} \cdot \boldsymbol{n} d S_{l}
$$

where

$S_{l}=$ the interfaces between the various compartments

$\sum_{l}=$ the summation over all interfaces

$\boldsymbol{r}^{\prime}=$ the point of observation on surface $S_{k}$

$\boldsymbol{r}=$ the source point at interface $S_{l}$

$\sigma_{s} \quad=$ the conductivity of the source region

$\bar{\sigma}_{k}=$ the average of the electrical conductivities at both sides of boundary $S_{\mathrm{k}}$

$\Delta \sigma_{l}=$ the conductivity just inside $S_{l}$ minus that just outside

$d \Omega_{k l}=$ the solid-angle distribution of surface $S_{l}$ seen from a point of observation on surface $S_{k}$

$\boldsymbol{n}=$ the outward normal at $\boldsymbol{r}$

$d S_{l}=$ the infinitesimal surface element on $S_{l}$

$V_{\infty}=$ the potential that arises from the primary sources only.

This formulation is known as the boundary element method.

The magnetic field distribution can be calculated from this potential distribution on the surfaces. For the magnetic field the formula, derived by GesElowitz (1970), holds:

$$
\boldsymbol{B}\left(\boldsymbol{r}^{\prime}\right)=\boldsymbol{B}_{\infty}\left(\boldsymbol{r}^{\prime}\right)-\frac{\mu_{0}}{4 \pi} \sum_{l} \Delta \sigma_{l} \int_{S_{l}} V_{l}(\boldsymbol{r}) \frac{\left(\boldsymbol{r}^{\prime}-\boldsymbol{r}\right)}{\left|\boldsymbol{r}^{\prime}-\boldsymbol{r}\right|^{3}} \times n d S_{l}
$$

where

$\boldsymbol{r}^{\prime}=$ the point of observation outside the volume conductor

$\boldsymbol{B}_{\infty}=$ the magnetic field that arises from the primary sources only.

Both integral equations for $V\left(\boldsymbol{r}^{\prime}\right)$ and $\boldsymbol{B}\left(\boldsymbol{r}^{\prime}\right)$ form the basis of our computational work. The Fredholm integral equation of the second kind (eqn. 1), must be solved to find the potential distribution on the surfaces $S_{k}$. When this problem is solved the magnetic field can be computed in a straightforward manner.

After decomposing the closed surfaces $S_{l}$ into $N_{l}$ trianglar elements (LYNN and TIMLAKE, 1968)

$$
S_{l}=\bigcup_{j=1}^{N_{l}} \Delta_{l}^{(j)}
$$

the surface integrals of eqn. 1 can be made discrete and eqn. 1 can be rewritten as the linear system

$$
\underline{V}=\underline{G}+\underline{\underline{B}} \cdot \underline{V}
$$

The elements of $V, G$ and $\underline{\underline{B}}$ are constant for all triangles and are defined as an average value over each triangle.

$$
\begin{aligned}
v_{k}^{(j)} & =\frac{1}{a_{k}^{(j)}} \int_{\Delta_{k}^{(j)}} V(\boldsymbol{r}) d S(\boldsymbol{r}) \\
g_{k}^{(j)} & =\frac{1}{a_{k}^{(j)}} \int_{\Delta_{k}(j)} G(\boldsymbol{r}) d S(\boldsymbol{r}) \\
b_{k l}^{(i j)} & =-\frac{1}{a_{k}^{(j)}} \frac{\Delta \sigma_{l}}{\bar{\sigma}_{k}} \int_{\Delta_{k}(j)} \Omega_{\Delta_{i, r^{(i)}}} d S(\boldsymbol{r})
\end{aligned}
$$

where

$\Omega_{\Delta_{i,} r^{(i)}}$ is $\frac{1}{2 \pi}$ times the solid angle subtended at $r^{\prime}$ by $\Delta_{i}^{(i)}$ and

$a_{k}^{(j)}$ is the area of triangle $\Delta_{k}^{(j)}$.

A straightforward solution of eqn. 3 is impossible as the matrix $B$ has an eigenvalue of one. Elimination of this singularity $(\lambda=1)$, as well as of some known eigenvectors with large eigenvalues $(|\lambda| \approx 1)$ from matrix $\underline{\underline{B}}$ has been carried out by using the deflation technique as described by LYNN and Timlake (1968).

Looking more closely at the original continuous Fredholm equation (eqn. 1), and its discrete version (eqn. 3), we see that the discrete version of the integral $\int_{s_{l}} V_{l} d \Omega_{k l}$ is the summation

$$
\sum_{j=1}^{N_{l}} V_{j} \Delta \Omega_{k j}
$$

The constancy of the potential $V_{j}$ over each triangle is considered to be an acceptable approximation as we have chosen a triangulation method such that all triangles involved subtend the same solid angle with respect to the location of the source. Even after this segmental refinement, the discrete potential distribution $V$ on surfaces $S_{k}$ may still differ from the continuous potential distribution $V_{k}\left(\boldsymbol{r}^{\prime}\right)$, as an error will be made in the discrete computation of the surface integrals. When the ratios of the conductivities at different interfaces $S_{k}$ is large, these errors can be magnified. The handling of this effect is the subject of this paper. An example of a spherical surface triangulated in the above mentioned way is given in Fig. 1.

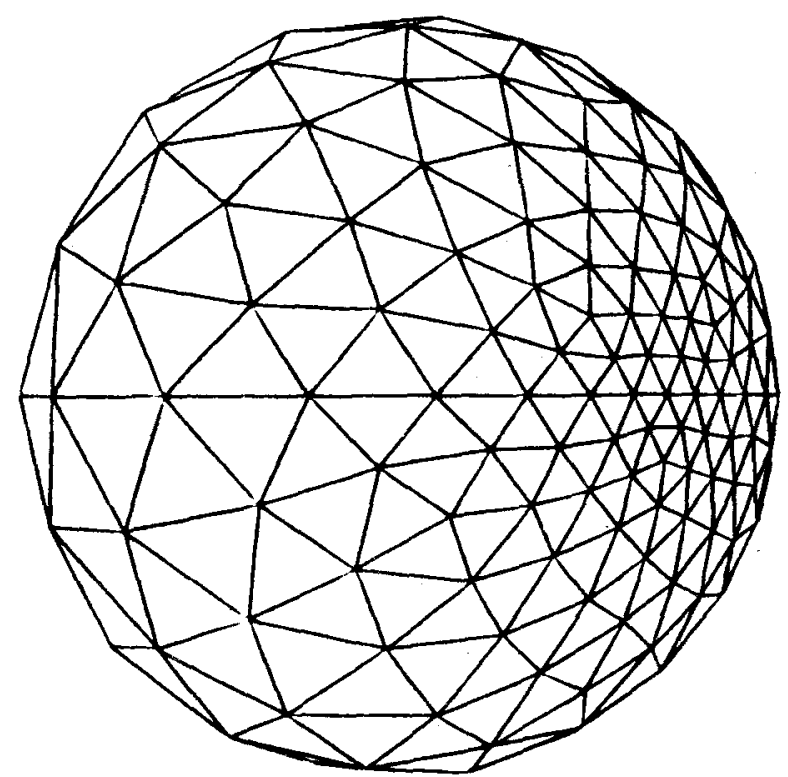

Fig. $1 A$ discrete representation of a sphere, with a segmental refinement such that the solid angles of all triangles involved, subtend the same solid angle with respect to the source location

\section{Two concentric spheres}

The errors which occur when using large conductivity ratios for the successive regions within a discrete multicompartment model of the head will now be analysed. We restrict ourselves to the simple model consisting of two concentric spheres, because for this configuration

(a) an analytic (exact) solution for the potential distribution is available

(b) the mathematical origin of the magnifying process of the discretisation error can be well demonstrated 
(c) numerical analysis of the error in the approximate solution can be carried out easily

(d) the effects of error-magnifying are essentially the same when a model consisting of more than two compartments is used.

For the two-sphere model (Fig. 2), we chose for $S_{1}$ and $S_{2}$ the respective surface boundaries of two concentric spheres both triangulated as depicted in Fig. 1.

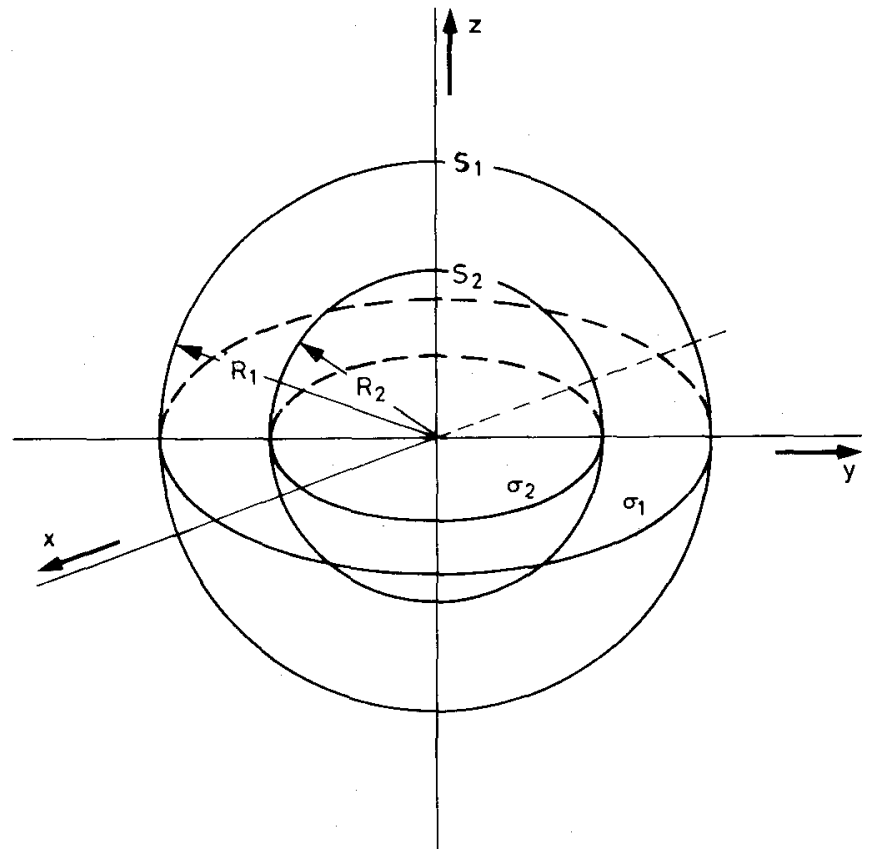

Fig. 2 Schematic representation of the two-sphere model in which $R_{1}=0.075 \mathrm{~m}$ and $R_{2}=0.063 \mathrm{~m}$. The source is located in $(x, y, z)=(0,0.05,0)$ in $m$

An excentrical located, tangential oriented dipole is chosen as a source, as for such a dipole the errors are expected to be greatest. When the potential distribution on the outer surface (equivalent to the EEG) is mapped we find a dipolar pattern. This consists of a line indicating zero value and at both sides, symmetrical to this line two extremes, namely a maximum and a minimum. Comparison of results based on either the analytical (exact) expression (CUFFIN and COHEN, 1979) or the numerical method based on eqn. 3 shows that in all maps obtained the position of the zero line and the extremes are correct. To evaluate the numerical error we can therefore restrict the error analysis by considering only the values of the extremes of the potential distribution.

The evaluation is carried out for the two spheres mentioned having radii $R_{1}=0.075 \mathrm{~m}$ and $R_{2}=0.063 \mathrm{~m}$. The distance of the tangentially oriented current dipole from the centre of the spheres is $0.05 \mathrm{~m}$. The source strength is $5 \times 10^{-6} \mathrm{Am}$. Choosing several values of the conductivity ratio $\sigma_{1} / \sigma_{2}$, within the system as described above, resulted in the set of data as listed in Table 1. $E$ is defined as the

\begin{tabular}{|c|c|}
\hline$\sigma_{1} / \sigma_{2}$ & $\begin{array}{c}E, \\
\text { per cent }\end{array}$ \\
\hline 100 & $2 \cdot 6$ \\
\hline 10 & 1.6 \\
\hline 1 & 1.8 \\
\hline $0 \cdot 1$ & $14 \cdot 5$ \\
\hline 0.05 & $30 \cdot 1$ \\
\hline 0.01 & 147.0 \\
\hline
\end{tabular}

relative error in the extremum on the outer surface:

$$
E=\left|\frac{V_{1, \text { extr }}(\text { num })-V_{1, \text { extr }}(\text { exact })}{V_{1, \text { extr }}(\text { exact })}\right|
$$

where

$V_{1, \text { extr }}($ exact) is the exact value of the extremum, found with the analytical expression and $V_{1, \text { extr }}($ num $)$ is the value of the extremum computed numerically using eqn. 3 .

With conductivities $\sigma_{1}$ and $\sigma_{2}$ in the successive compartments, eqn. 1 reads

$$
\begin{aligned}
V_{1}= & \frac{2 \sigma_{s}}{\sigma_{1}} V_{\infty}-\frac{2 \sigma_{1}}{\sigma_{1}} \frac{1}{4 \pi} \int_{s_{1}} V_{1} d \Omega_{11} \\
& -\frac{2\left(\sigma_{2}-\sigma_{1}\right)}{\sigma_{1}} \frac{1}{4 \pi} \int_{s_{2}} V_{2} d \Omega_{12} \\
V_{2}= & \frac{2 \sigma_{s}}{\sigma_{1}+\sigma_{2}} V_{\infty}-\frac{2 \sigma_{1}}{\sigma_{1}+\sigma_{2}} \frac{1}{4 \pi} \int_{S_{1}} V_{1} d \Omega_{21} \\
& -\frac{2\left(\sigma_{2}-\sigma_{1}\right)}{\sigma_{1}+\sigma_{2}} \frac{1}{4 \pi} \int_{s_{2}} V_{2} d \Omega_{22}
\end{aligned}
$$

When $\sigma_{1} / \sigma_{2} \ll 1$ it follows from eqns. 5 and 6 that the coefficients of the 'auto'-integrals $\int V_{1} d \Omega_{11}$ and $\int V_{2} d \Omega_{22}$ have the same order of magnitude.

The coefficient of $\int V_{1} d \Omega_{21}$ is much smaller than the coefficent of $\int V_{1} d \Omega_{11}$.

The high value of the coefficent of $\int V_{2} d \Omega_{12}$ actually causes magnification of the error made in the numerical computation of this integral. When, for example, the integral $\int V_{2} d \Omega_{12}$ is calculated to an accuracy of 1 per cent (which can be expected), the error in the second term of the right hand side of eqn. 6 will be blown up by a factor $2 \sigma_{2} / \sigma_{1}$ due to the influence of the coefficient as all values of $V$ are of the same order of magnitude.

If $\sigma_{1} / \sigma_{2}>1$, all coefficients involved would be of the same order of magnitude or smaller than the coefficients of the 'auto'-integrals. No error-magnification has to be expected in this case. From this argumentation the data as listed in Table 1 can be understood.

We have to find a method to eliminate the error magnification when using a realistical chosen conductivity ratio of the order of $\sigma_{1} / \sigma_{2}=0 \cdot 01$, which is much smaller than 1 .

The value of the potential in the extremum on the outer surface resulting from the numerical solution method of eqn. 3 depends on the total number of discrete points $N$. Therefore, this value is called $V_{1, \text { extr }}^{N}($ num $)$.

From the results presented in Table 2 it can be easily extrapolated that for reaching a final error of 5 per cent in $V_{1, \text { extr }}^{N}($ num $)$ the number of discrete points $N$ has to be of the order of $10^{4}$. The minimal amount of computational work for the iterative solution of eqn. 3 is proportional to $N^{2} \log N$ (Schippers, 1982). For this case this dimensionless number will be of the order of $4 \times 10^{8}$. For the DEC 20 computer which is at our disposal, this number is

Table 2 Errors as a function of the number of discrete points for the two-sphere system

\begin{tabular}{rcccc} 
& & & $E$ & \\
$N$ & $\begin{array}{c}V_{1, \text { extr }}^{N}(\text { num }), \\
\mathrm{mV}\end{array}$ & $\begin{array}{c}E, \\
\mathrm{mV}\end{array}$ & $\begin{array}{c}V_{1, \text { extr }} \text { (exact) } \\
\text { per cent }\end{array}$ & $\begin{array}{c}N \times E, \\
\mathrm{mV}\end{array}$ \\
\hline 84 & 8.92 & 7.60 & 576 & 638 \\
160 & 5.33 & 4.01 & 304 & 642 \\
324 & 3.27 & 1.95 & 147 & 632 \\
640 & 2.32 & 1.00 & 75 & 640 \\
\hline
\end{tabular}

The analytical found exact solution $V_{1, \text { extr }}($ exact $)$ is $1.32 \mathrm{mV}$ 
equivalent to a CPU-time of $3 \mathrm{~h}$ for one iteration of eqn. 3 . Fortunately, the amount of computational work can be decreased substantially by means of the Richardson extrapolation technique.

\section{Richardson extrapolation}

We can expand the error in the approximate solution in a power series of $N_{l}$ as follows:

$$
\int_{S_{l}} V_{l} d \Omega_{k l}-\sum_{j=1}^{N_{l}} V_{j} \Delta \Omega_{k j}=\frac{1}{N_{l}} \varepsilon_{1}+\frac{1}{N_{l}^{2}} \varepsilon_{2}+\cdots
$$

where

$N_{t}$ is the number of triangles on surface $S_{l}$ and $\varepsilon_{i}$ does not depend on $N_{l}$.

The Richardson extrapolation procedure may be used when the error satisfies the condition (RICHARDSON and GUANT, 1927)

$$
\left|\frac{1}{N_{l}} \varepsilon_{1}\right| \gg\left|\frac{1}{N_{l}^{2}} \varepsilon_{2}+\frac{1}{N_{l}^{3}} \varepsilon_{3}+\cdots\right|
$$

If this condition is met, eqn. 7, taking two discrete levels $N_{1}$ and $N_{2}$ on surface $S_{l}$, reads:

$$
\begin{aligned}
& \int_{S_{l}} V_{l} d \Omega_{k l}-\sum_{j=1}^{N_{1}} V_{j} d \Omega_{k j}=\frac{1}{N_{1}} \varepsilon_{1} \\
& \int_{S_{l}} V_{l} d \Omega_{k l}-\sum_{j=1}^{N_{2}} V_{j} d \Omega_{k j}=\frac{1}{N_{2}} \varepsilon_{1}
\end{aligned}
$$

Elimination of $\varepsilon_{1}$ from eqns. 9 and 10 results in

$$
\int_{S_{l}} V_{l} d \Omega_{k l}=\frac{N_{1}}{N_{1}-N_{2}}\left(\sum_{j=1}^{N_{1}} V_{j} \Delta \Omega_{k j}-\frac{N_{2}}{N_{1}} \sum_{j=1}^{N_{2}} V_{j} \Delta \Omega_{k j}\right)
$$

This means that with the help of two numerical approximations, the value of the integral $\int_{S_{l}} V_{l} d \Omega_{k l}$ can be obtained.

The potential in eqn. 1 on the surfaces $k$ consists (apart from the exact term $\left.\sigma_{s} / \bar{\sigma}_{k} V_{\infty}\left(\boldsymbol{r}^{\prime}\right)\right)$ of a summation of integrals, all with weighting factors independent of $N_{l}$. We choose the number of discrete points to be the same on all surfaces, $N_{l}$. The total number of discrete points on $n$ surfaces will be $N=n N_{l}$. One can easily derive that when the Richardson condition (eqn. 8) is met for the integration over one surface, the total error $E$ will satisfy the Richardson condition.

In Table 2 we see that $N \times E$ has approximately the same value for all numbers of $N$. This means that in the case of two spheres the condition necessary for Richardson extrapolation is met and that the exact value of the potential can be obtained from two numerical approximations based on two different discretisation levels. Using the Richardson extrapolation procedure for the two-sphere model, based on the discretisation levels $N_{1}=324$ and $N_{2}=640$, resulted in an error in the final potential distribution which was less than 2 per cent. The computational work necessary for the iterative solution is of the order $0\left((324)^{2} \log 324+(640)^{2} \log 640\right)=0\left(1.4 \times 10^{6}\right)$ per iteration. This is a factor 285 times faster than without the use of the Richardson extrapolation procedure.

\section{Numerical procedures}

To perform the Richardson extrapolation procedure we have to construct two distinct discretisation levels and perform two successive iterations, one at each level. As we have described our surfaces with plane triangular elements (see Fig. 1), both the vertex points and the centres of mass of the triangles can be used as discrete points. This means that the same triangulation yields, respectively, $N_{l}$ and $\left(2 N_{l}-4\right)$ discrete points on each surface.

The centres of mass have been used as discrete points initially in the numerical solution of eqn. 3 (LYNN and TIMLAKE, 1968). The numerical procedure is identical if the vertices are chosen as discretisation points although the solid-angle terms $\Delta \Omega_{k l}$ (and especially $\Delta \Omega_{k k}$ ) have to be re-evaluated.

To speed up the numerical process in the centres of mass approach, the interpolated final potential vector resulting from the vertex approach can be used as the starting vector of the iterative process. By doing so, the number of iterations needed in the centres of mass approach can be reduced by 20 per cent.

\section{Results}

As shown in this paper the numerical solution of the forward problem for EEGs can be speeded up substantially by using the Richardson extrapolation technique. To use this extrapolation technique the error has to be inversely proportional to the total number of discrete points. We demonstrated that for a model of the head consisting of two concentric spheres this condition is met.

Also in the more general case of a model consisting of four concentric spheres, the Richardson condition is met. Using the same error analyses as introduced for the twosphere model, we found for the four-sphere model results as listed in Table 3. The radii of the four spheres were adapted to conform to the head and were, respectively, $0.075,0.071,0.065$ and $0.063 \mathrm{~m}$. The conductivities of the compartments were chosen to be $0.33,0.0042,1.00$ and $0.33 \mathrm{Sm}^{-1}$. These values correspond with those reported elsewhere (GEDDES and BAKER, 1967) and represent, respectively, the conductivities of the scalp, the skull, the CSF and the brain tissue.

We verified that the Richardson condition is also met when the shape of the compartment boundaries within the head are chosen more realistically (MeIJs et al., 1985). This implies that the numerical methods described in this paper can be used for treating all multicompartment models of the electrical volume conductor decribing the head.

\section{Discussion}

The strategy of using the Richardson extrapolation technique to reduce computational errors, resulting from an ill-conditioned Fredholm integral equation of the second kind, has been followed in other fields of computational research (SATHIARAJ and SANKaR, 1983; Dobrovol'SkII, 1981). Until now, it does not seem to have been applied to the numerical problem discussed here. This can be

\begin{tabular}{|c|c|c|c|c|}
\hline \multirow[b]{2}{*}{$N$} & \multirow[b]{2}{*}{$\underset{\mathrm{i}, \text { extr }}{V^{N}}($ num $)$} & \multirow[b]{2}{*}{$\begin{array}{c}E \\
\mathrm{mV}\end{array}$} & $E$ & \multirow[b]{2}{*}{$\begin{array}{c}N \times E, \\
\mathrm{mV}\end{array}$} \\
\hline & & & $\overline{V_{1, \text { extr }}(\text { exact })}$, & \\
\hline 168 & $2 \cdot 81$ & $2 \cdot 25$ & 402 & 378 \\
\hline 320 & 1.79 & $1 \cdot 23$ & 220 & 394 \\
\hline 648 & $1 \cdot 13$ & 0.57 & 102 & 370 \\
\hline 1280 & 0.86 & 0.30 & 54 & 384 \\
\hline
\end{tabular}
explained by the fact that the conductivity ratios in discrete torso models are usually small and so the need for it

Table 3 Errors as a function of the number of discrete points for the four-sphere system

For this configuration the analytical found exact solution $V_{1, \text { extr }}($ exact $)$ is $0.56 \mathrm{mV}$ 
is absent. However, using a discrete model of the head implies that the conductivities in the successive compartments are such that the errors will be magnified. Richardson's extrapolation technique was shown to adequately handle this problem.

\section{References}

Barnard, A. C. L., Duck, J. M., LynN, M. S. and Timlake, W. P. (1967) The application of electromagnetic theory to electrocardiology II. Biophys. $J ., 7,463-491$.

Barth, D. S., Sutherling, W., Engle, J. Jr., and Beatty, J. (1984) Neuromagnetic evidence of spatially distributed sources underlying epileptiform spikes in the human brain. Science, 223, 293-296.

Cohen, D. and Cuffin, B. N. (1983) Demonstration of useful differences between magnetoencephalogram and electroencephalogram. Electroenceph. Clin. Neurophysiol., 56, 38-51.

CufFin, B.N. and CoHEN, D. (1979) Comparison of the magnetoencephalogram and electroencephalogram. Ibid., 47, 131-146.

DoBrovol'sKII, I. P. (1981) Richardson extrapolation in the approximate solution of Fredholm integral equations of the second kind. USSR Comput. Maths. \& Math. Phys., 21, (4), 139-149.

GedDEs, L. A. and BAKER, L. E. (1967) The specific resistance of biological material, a compendium of data for the biological engineer and physiologist. Med. \& Biol. Eng., 5, 271-293.

Geselowitz, D. B. (1970) On the magnetic field generated outside an inhomogeneous volume conductor by internal sources. IEEE Trans. Mag-6, 346-347.

Lynn, M. S. and Timlake, W. P. (1968) The use of multiple deflations in the numerical solution of singular systems of equations with applications to potential theory. Siam. $J$. Numer. Anal., 5, 303-322.

Meiss, J. W. H., Peters, M. J. and van Oosterom, A. (1985) Computation of MEGs and EEGs using a realistically shaped multicompartment model of the head. Med. \& Biol. Eng. \& Comput., 23, suppl. part 1, 36-37.

OKADA, Y, (1983) Brain studies. In Biomagnetism: an interdisciplinary approach. Williamson, S. J., Romani, G. L., KaUfmaN, L. and Modena, I. (Eds.) Plenum Press, New York, 417-426.

Peters, M. J., Swennenhuis, M. J. M., van Oosterom, A. and WeVERS-HenKe, J. J. (1983) The influence of inhomogeneities on the cardiac-magnetic-field distribution. Il Nuovo Cimento 2D, (N2), 324-339.

Ricci, G. B., Leoni, R., Romani, G. L., Campitelli, F., Buonomo, S. and ModenA, I. (1984) 3-D Neuromagnetic localization of sources of interictal activity in cases of focal epilepsy. In Biomagnetism: applications \& theory. WeINBERG, H., STroINK, G. and Katila, T. (Eds.) Pergamon Press, 304-310.

Richardson, L. F. and Guant, J. A. (1927) The deferred approach to the limit. Phil. Trans. R. Soc., 226, 299-361.

Romani, G. L., Leoni, R. and Salustri, C. (1985) Multichannel instrumentation for biomagnetism. In SQUID 85, НAHLBOHM, H. D., and LüBBIG, H. (Eds.) de Gruytes, 919-932.

SathiaraJ, D. D. and Sanker, R. (1983) The method of successive extrapolated iterated defect correction and its application to second kind Fredholm's integral equation. BIT (Copenhagen), 23, (2), 239-247.

SCHIPPERS, H. (1982) Application of multigrid methods for integral equations to two problems from fluid dynamics. J. Comp. Phys., 48, 441-461.

Sutherling, W., Barth, D. S. and Beatty, J. (1984) Magnetic fields of epileptic spike foci: equivalent localization and propagation. In Biomagnetism: applications \& theory. WEINBERG, $\mathrm{H}$., Stroink, G. and Katila, T. (Eds.) Pergamon Press, 249-260.

\title{
Technical note
}

\section{Low-cost Doppler signal simulator}

\author{
C. D. Sheldon \\ T. C. Duggen \\ Department of Clinical Physics \& Bio-Engineering, 11 West Graham Street, \\ Glasgow G4 9LF, UK
}

Keywords-Doppler ultrasound, Mean frequency follower, Spectral analysis

Med. \& Biol. Eng. \& Comput., 1987, 25, 226-228

\section{Introduction}

THE INCREASING sophistication of Doppler ultrasound systems makes it difficult to evaluate their performance. Many compute the instantaneous maximum or mean frequency and use this to obtain the flow (ARTS and RoEVRos, 1972), estimate the pressure gradient (HATLE et al., 1978), or measure parameters such as pulsatility index (JOHNSTON et al., 1984). Often, this computation is performed by analogue devices, the operating characteristics of which have not been established for different spectral shapes.

The shape of the instantaneous amplitude against frequency Doppler spectrum depends upon the velocity

First received 24th January and in revised form 28th July 1986

(C) IFMBE: 1987 profile in the vessel (GiLL, 1979) and the geometrical relationship between the ultrasound field and the vessel under investigation (Evans, 1982). Large variations in the shape of the instantaneous Doppler spectrum have been demonstrated both in vitro (KALMAN et al., 1985) and in vivo (SHELdon et al., 1983). A simple, stable and low-cost method of simulating these Doppler spectra is described and its use is illustrated by obtaining the operating characteristics of a commercial mean frequency follower.

\section{Method}

A block diagram of the Doppler simulator is shown in Fig. 1. A National Semiconductor MM5837 digital noise source, based on a pseudorandom sequence generator, is 